\title{
An investigation into the deformation, movement and coalescence characteristics of water-in-oil droplets in an AC electric field
}

\author{
He Li-min ${ }^{1 *}$, Yang Dong-hai ${ }^{1}$, Gong Rong-na ${ }^{2}$, Ye Tuan-jie ${ }^{3}$, LÜ Yu-ling ${ }^{1}$ and \\ Luo Xiao-ming ${ }^{1}$ \\ ${ }^{1}$ College of Pipeline and Civil Engineering, China University of Petroleum, Qingdao, Shandong 266580, China \\ ${ }^{2}$ Beijing Petrochemical Engineering Co., Ltd. Beijing 100107, China \\ ${ }^{3}$ Petro China Oil \& Gas Pipeline Control Center, Beijing 100007, China
}

(C) China University of Petroleum (Beijing) and Springer-Verlag Berlin Heidelberg 2013

\begin{abstract}
Drop-drop coalescence is important in electric dehydrators used for oil-water separation in the oil industry. The deformation degree, angle between the electric field and the center line of two drops, effects of intensities and frequencies of the electric field have been studied by analyzing droplet images. However, seldom have people investigated the movement and the relative velocity in the process of dropdrop coalescence. In this paper forces acting on a single droplet and horizontal water droplets in an AC electric field were analyzed, and experiments were carried out to investigate the deformation, movement and coalescence characteristics of droplets with white oil and water. With a micro high-speed camera system and image processing technology, the droplet images were collected and analyzed. The results indicate that the deformation is mainly affected by the electric field intensity, frequency, droplet diameter and the oil viscosity. High field strength and large diameter facilitate deformation of drops in the electric field. The effect of frequency and oil viscosity is not obvious. Higher frequency and higher oil viscosity will lead to smaller oscillation amplitude. The effect of electric field intensity and droplet diameter on oscillation amplitude is not obvious. When the center-to-center distance between droplets is large, the forces acting on droplets in the horizontal direction are mainly dipole-dipole attraction and drag forces. There is also the film-thinning force when droplets get closer. The forces are simplified and derived. Based on force analysis and Newton's second law, the relative movement is analyzed in different parts, and the relationship of center-to-center distance and time is in accordance with an explinear function at different stages. According to experimental data, the movement of $145 \mu \mathrm{m}$ double droplets before coalescence can be fitted well with an explinear function at two stages. In addition, the whole movement process is investigated and can be estimated with a fourth order polynomial curve, from which the relative velocity of droplet movement can also be obtained. With an increases in electric field intensity and droplet diameter and a decrease in oil viscosity, the relative velocity increases. Only when the oil-water interfacial tension is obviously high, can it influence the relative movement significantly. The coalescence is mainly dipole coalescence and chain coalescence under influence of the AC electric field.
\end{abstract}

Key words: Deformation, coalescence, velocity, AC electric field

\section{Introduction}

Dehydration of water in oil (W/O) emulsion becomes difficult as the crude oil properties change continuously with oil production, especially when a large amount of polymer is added to enhance oil production. Historically, there are several available methods, such as chemical demulsification, gravity or centrifugal settling, heat treatment and electrostatic demulsification (Feng and Guo, 2006). Separation methods

*Corresponding author: email: helimin@vip.163.com

Received June 15, 2013 utilizing high electric fields have been used extensively in petroleum industry (Eow et al, 2003). Most of the conventional electro-separators are huge and bulky, thus having high capital and operating costs. A compact electrocoalescer will have the advantage of being smaller and lighter than traditional ones and thus easier to install on an offshore platform and reduce the investment needed. There is, therefore, great scope to optimize the design and operation of these separators based on a fundamental understanding of parameters that affect the coalescence of aqueous drops in oil. However, the fundamental mechanisms governing the behavior of water in oil emulsion under a high electric 
field are still not very well understood, due to the complex interaction among various influencing factors.

When droplets are exposed to an AC electric field, the droplets can be elongated to different shapes. Droplets can also be charged by induction by the interaction between the electric field and the drops. Two nearby droplets attract each other as the charge polarity on adjacent surfaces is opposite. Due to the electrically induced force, the two droplets move together and then coalesce. Under high electric field intensity, the relative speed of two droplets is increased. Two droplets collide which can lead to great damage to the interfacial film between two droplets. The film can be ruptured and coalescence occur. In the AC electric field the oscillation of droplets can also damage the film.

Researchers have paid attention to droplet deformation and coalescence for a long time. With the development of low-cost high speed cameras, studying the deformation and movement of micro droplets is now much more practical. Eow and Ghadiri (2003) investigated the deformation of aqueous drops in several liquid/liquid systems under a DC electric field with a high speed camera. The diameter of the drops was about $1 \mathrm{~mm}$. The effect of electric field intensity and interfacial tension were analyzed. Before rupture, the droplet is elongated and the deformation degree is proportional to the electric field intensity. The degree of deformation is different in different liquid/liquid systems, depending on the physical properties of the respective systems. Eow et al (2001) and Eow and Ghadiri (2003b) also found the shape of drops is related to conductivity, viscosity of the continuous phase, interfacial tension and viscosity of the drop liquid. The deformation degree is small under a small dielectric constant of oil and large interfacial tension. Supeene et al (2008) explored the influence of the fluid, interfacial, and electrical properties on the system dynamics by a numerical method. The simulations were compared with analytic solutions, as well as available experimental results, which indicated that the predictions from the model were reliable even at considerably large deformations.

The relative movement of two droplets in an electric field is governed by the forces acting on the droplets. Bails and Larkai (1981) and Taylor (1996) indicated that fieldinduced charges on the water droplets cause adjacent droplets to attract each other. The electrically induced force was the most important force to promote droplet coalescence. Williams (1989) ignored the deformation of polarized droplets in the electric field, and calculated the electrically induced force between double droplets based on the spherical drop polarization model. Eow did a lot of work on droplet coalescence. Eow and Ghadiri $(2001,2002)$ reviewed the development of dehydrating technology and the development of investigation into droplet coalescence. Eow and Ghadiri (2001) pointed out that under a high strength electric field or when the center-to-center distance of two droplets was short, the result calculated with the spherical droplet polarization model was not accurate. Eow and Ghadiri (2003a) investigated the effects of the applied electric field, angle between the electric field and the center line of the two drops, and also electrode geometry to droplet coalescence under a pulsed electric field. Eow et al (2007) also studied droplet coalescence in a new dehydrator. Chiesa et al (2005a, 2005b, 2006) analyzed all the forces acting on water droplets and simulated their movement process numerically. Also, they investigated the coalescence process of a small droplet falling onto a stationary bigger one experimentally, and compared the experimental data with the numerical simulation results. A lot of papers also focus on the effect of electric field intensity and frequency on the coalescence efficiency of the dehydrator (Zhang et al, 2011; Harpur et al, 1997; Urdahl et al, 1998).

Seldom did people investigate the approaching process and relative speed of two droplets in an AC electric field. The main emphasis of the present work is placed on investigating the movement of two horizontal droplets before coalescence in an AC electric field, especially the change of centerto-center distance and relative speed. The expressions of different forces have been simplified and derived. Finally, the micro-experimental data and numerically predicted results were compared and analyzed.

Williams and Bailey (1996) believed dipole-dipole coalescence and electrophoresis lead to drop coalescence under the electric field. Bailes and Stitt (1987) believed that dipole coalescence is the main reason for droplet coalescence in AC and DC electric fields. Bailes and Larkai (1982) published a theory that dielectric relaxation was important during droplet coalescence and before coalesce droplets form chains. Chen et al (1994) also found in experiments that droplet chains are short in a high frequency field while long in a low frequency field. Taylor (1988) pointed that when an $\mathrm{AC}$ electric field is applied very stable droplet chains form between the two electrodes, which can cause current leakage and prevent droplet coalescence.

There is little published on the coalescence of droplets less than $100 \mu \mathrm{m}$, and seldom did people investigate the approaching process and relative speed of two droplets in an AC electric field. The main emphasis of the present work was placed on investigating the deformation of single droplets, movement of two horizontal droplets and droplet coalescence in the AC electric field, especially the changes of centerto-center distance and relative speed. The expressions of different forces have been simplified and derived. Finally, the micro-experimental data and numerically predicted results were compared and analyzed.

\section{Theory}

\subsection{Forces applying on a single drop}

Forces on a droplet are shown in Fig. 1. The forces acting on a droplet include electric force $F_{\mathrm{e}}$, additional stress caused by interfacial tension $\Delta p$, gravity $G$, buoyancy force $F_{\mathrm{b}}$, drag force $F_{\mathrm{d}}$, and virtual mass force $F_{\mathrm{a}}$. When surrounded by oil, both ends of the drop have different charges induced, like a dipole. Thus, the drop will be elongated by the electric force to an ellipsoidal shape, which is shown in Fig. 1(b). The electric field intensity is $E$ in the continuous phase. The relative dielectric constant is $\varepsilon_{\mathrm{c}}$. Assuming the coordinate of a point in the sphere is $B(x, y, z)$, and the angle between the direction of the electric field and the radius to point $B$ is $\zeta$. Therefore, the induced surface charge density at point $B, \varphi_{B}$ (Guo, 2001) is: 


$$
\phi_{B}=3 \varepsilon_{0} \varepsilon_{\mathrm{c}} E \cos \zeta
$$

where $\varepsilon_{0}$ is the permittivity of vacuum, its value is $8.8542 \times 10^{-12}$ $\mathrm{F} / \mathrm{m}$. The electric force $F_{\mathrm{e}}$ of $\Delta S$ is:

$$
F_{\mathrm{e}}=\phi_{B} E \Delta S=3 \varepsilon_{0} \varepsilon_{\mathrm{c}} E^{2} \Delta S \cos \zeta
$$



(a) Forces acting on a drop

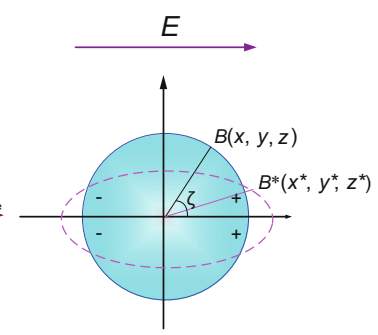

(b) Deformation of a drop
Fig. 1 Forces and deformation of a single drop in a uniform electric field

From Eq. (2), it can be found the maximum values of the surface charge density exist where $\zeta$ is 0 and $\pi$. After applying the electric field, the drop will deform into an ellipsoid as the electric force on the surface is nonuniform. The surface charge density is proportional to the curvature, so the surface charge density is a maximum at the two ends of the ellipsoid. The eccentricity of the ellipsoid $e$, additional stress $\Delta p$ and drag force $F_{\mathrm{d}}$ are shown as follows:

$$
\begin{aligned}
& e=\sqrt{1-\frac{b^{2}}{a^{2}}} \\
& \Delta p=\frac{2 \gamma}{r_{0}} \\
& F_{\mathrm{d}}=C_{\mathrm{D}} \frac{v^{2}}{2} \rho A_{\mathrm{p}}
\end{aligned}
$$

where $a$ and $b$ are the long axis and the short axis of the ellipsoid, $\mathrm{m} ; r_{0}$ is the initial radius of the droplet, $\mathrm{m} ; \gamma$ is the interfacial tension, $\mathrm{N} / \mathrm{m} ; C_{\mathrm{D}}$ is the drag coefficient; $A_{\mathrm{p}}$ is the projected area of the sphere, $\mathrm{m}^{2} ; \rho$ is the density of the continuous phase, $\mathrm{kg} / \mathrm{m}^{3}$. In this paper $C_{\mathrm{D}}=\frac{24}{R e}$.

\subsection{Forces acting on two droplets}

In this paper, in order to facilitate the theoretical analysis, several assumptions are made as follows: 1) In the microexperiments, the deformation degrees of all the dispersed water droplets, the diameters of which are smaller than 200 $\mu \mathrm{m}$, are so small in the applied electric field that the dispersed droplets can be considered as rigid. 2) The edge effects of the plate type electrode is ignored, so it is assumed that the electric field is uniform. 3) Compared to the horizontal movement of water droplets during the experiment, the movement in the vertical direction can be ignored, so only the forces acting on the droplets in the horizontal direction were analyzed.

The forces acting on spherical double droplets of the same diameter in the applied electric field are shown in Fig. 2. The radii of the both droplets are $r_{1}$ and $r_{2}$. Here, $r=2 r_{1} r_{2} /\left(r_{1}+r_{2}\right)$ is defined as the reduced radius. When $r_{1}=r_{2}, r=r_{1}=r_{2} . d$ is the center-to-center distance between the two droplets. The gap between the surfaces of two droplets is $h$, then $d^{\prime}=d /(2 r)$ is defined as the relative center-to-center distance between the two droplets. The applied electric field intensity is known as $E$.

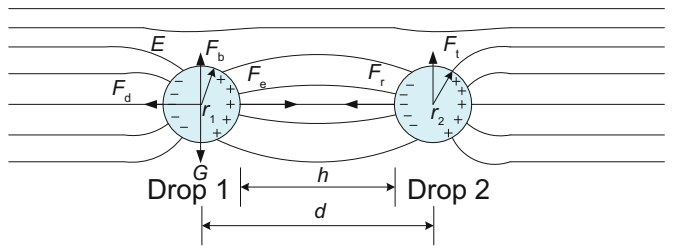

Fig. 2 Charge distribution and forces on droplets in the electric field

Taking droplet 1 for example, it moves to the right, and its trajectory can be calculated by integrating Newton's second law as follows:

$$
\begin{aligned}
& \frac{\mathrm{d} \vec{x}_{1}}{\mathrm{~d} t}=\vec{v}_{1} \\
& m_{1} \frac{\mathrm{d} \vec{v}_{1}}{\mathrm{~d} t}=\vec{F}_{\text {fluid }}+\vec{F}_{\text {ext }}+\vec{F}_{\text {d-d }}
\end{aligned}
$$

where $\overrightarrow{x_{1}}, m_{1}$ and $\overrightarrow{v_{1}}$ are the position, mass and velocity of droplet $1 ; \vec{F}_{\text {fluid }}$ represents the vector of forces acting from the fluid on the droplet $1 ; \vec{F}_{\text {ext }}$ is the external force; and $\vec{F}_{\text {d-d }}$ represents the droplet-droplet force. The modeling framework proposed by Chiesa et al (2005b) is employed in this paper.

\subsubsection{Modeling the fluid-droplet force $\vec{F}_{\text {fluid }}$}

$\vec{F}_{\text {fluid }}$ represents the vector of forces acting from the fluid on droplet 1 , which is modeled by summing the drag force $\vec{F}_{\text {d }}$, the virtual mass force $\vec{F}_{\text {vm }}$, and the buoyancy force $\vec{F}_{\mathrm{b}}$.

As is shown in Fig. 2, when there is a relative movement between the droplet and the continuous fluid, there is a drag force $\vec{F}_{\text {d }}$ acting from the fluid on the droplet in the opposite direction of its movement. In this work, the effect of internal circulation in the dispersed droplet on the drag force is ignored, and then the expression of the drag force $\vec{F}_{\mathrm{d}}$ is described as Eq. (5).

The virtual mass force $\vec{F}_{\text {vm }}$ represents the acceleration of the continuous fluid which is caused by the motion of the dispersed droplet when the fluid and the droplet have a relative acceleration (Chiesa et al, 2005b). In this paper, the continuous phase is stationary, and there is no acceleration, so $\vec{F}_{\text {vm }}=0$.

The buoyancy force $\vec{F}_{\mathrm{b}}$ is in the opposite direction to gravity, which can be expressed as:

$$
\vec{F}_{\mathrm{b}}=-\rho_{\mathrm{c}} g V_{\mathrm{d}} \vec{e}_{\mathrm{g}}
$$

where $V_{\mathrm{d}}$ is the droplet volume; and $\vec{e}_{g}$ represents the gravity 
vector.

\subsubsection{Modeling the external force $\vec{F}_{\text {ext }}$}

As is shown in Fig. 2, the only external force acting on a dispersed droplet is gravity $G$, expressed as follows:

$$
\vec{G}=\rho_{\mathrm{d}} g V_{\mathrm{d}} \overrightarrow{e_{g}}
$$

\subsubsection{Modeling droplet-droplet force $\vec{F}_{\text {d-d }}$}

The droplet-droplet force in the applied electric field mainly consists of the film-thinning force $\vec{F}_{\mathrm{f}}$, due to the drainage of the fluid between the droplets, and the electric force $\vec{F}_{\text {e }}$ due to the difference in polarization over the droplet interface.

The strength of the interfacial film between water droplets is crucial to the water-oil emulsion, and the lifetime of the liquid film is governed by the film-thinning and rupture processes. The film-thinning force, which is caused by the drainage of the liquid film between the two neighboring droplets, can prevent two droplets from touching each other. The derivation of $\vec{F}_{\mathrm{f}}$ requires that the gap between the droplets are very small, $h<<r$, and here $r$ represents the reduced radius. For rigid liquid droplets, the expression of the film-thinning force (Davis et al, 1989) is shown as:

$$
\vec{F}_{\mathrm{f}}=\frac{6 \pi \mu_{\mathrm{c}} r^{2}\left(\overrightarrow{v_{\mathrm{r}}} \cdot \overrightarrow{e_{\mathrm{r}}}\right)}{h} \vec{e}_{\mathrm{r}}
$$

where $\mu_{\mathrm{c}}$ represents the viscosity of the continuous phase, $\overrightarrow{v_{\mathrm{r}}}$ and $\vec{e}_{\mathrm{r}}$ are the relative velocity vector and the direction of the relative motion, respectively.

The dipole-dipole force $\vec{F}_{\text {e }}$ between two droplets can be divided into two parts: the radial force $\vec{F}_{\mathrm{r}}$ and the tangential force $\vec{F}_{\mathrm{t}}$. There are two different models for calculating the droplet-droplet electrically induced force. For a large droplet gap $h>>r$, the electrically induced force is only affected by the polarization of droplets, and the electrostatic interaction between two droplets can be approximated as the force between two dipoles located at the spherical droplet centers, then point-dipole model is chosen to calculate $\vec{F}_{\text {e }}$. For small separations $h<r$, the size of water droplets cannot be ignored, and can influence the droplet-droplet electrically induced force, then dipole-induced-dipole model is applied to calculated $\vec{F}_{\mathrm{e}}$.

The expression of point-dipole model (Klingenberg et al, 1991) is:

$$
\begin{aligned}
& \vec{F}_{\mathrm{r}}=12 \pi \beta^{2} \varepsilon_{\mathrm{o}}|E|^{2} r_{2}^{3} r_{1}^{3}|d|^{-4}\left(3 \cos ^{2} \theta-1\right) \\
& \vec{F}_{\mathrm{t}}=-12 \pi \beta^{2} \varepsilon_{\mathrm{o}}|E|^{2} r_{2}^{3} r_{1}^{3}|d|^{-4} \sin 2 \theta
\end{aligned}
$$

with

$$
\beta=\frac{\varepsilon_{\mathrm{w}}-\varepsilon_{\mathrm{o}}}{\varepsilon_{\mathrm{w}}+2 \varepsilon_{\mathrm{o}}}
$$

where $\theta$ represents the angle between the center line of droplets and the direction of the applied electric field. In the present work $\theta=0^{\circ}$.
For a large droplet distance, there is no film-thinning force, and the droplet-droplet force $\vec{F}_{\mathrm{d} \text {-d }}$ only includes the electrically induced force $\vec{F}_{\mathrm{e}}$ (point-dipole model), and can be expressed:

$$
F_{\mathrm{d}-\mathrm{d}}=F_{\mathrm{r}}=24 \pi \beta^{2} \varepsilon_{\mathrm{o}}|E|^{2} r_{2}^{3} r_{1}^{3}|d|^{-4}
$$

The expression of the dipole-induced-dipole model (Siu et al, 2001) is indicated as follows:

$$
\begin{aligned}
& \vec{F}_{\mathrm{r}}=12 \pi \beta^{2} \varepsilon_{\mathrm{o}}|E|^{2} r_{2}^{3} r_{1}^{3}|d|^{-4}\left(3 K_{1} \cos ^{2} \theta-1\right) \\
& \vec{F}_{\mathrm{t}}=-12 \pi \beta^{2} \varepsilon_{\mathrm{o}}|E|^{2} r_{2}^{3} r_{1}^{3}|d|^{-4} K_{2} \sin 2 \theta
\end{aligned}
$$

with

$$
\begin{aligned}
& K_{1}=1+\frac{\beta r_{1}^{3}|d|^{5}}{\left(|d|^{2}-r_{2}^{2}\right)^{4}}+\frac{\beta r_{2}^{3}|d|^{5}}{\left(|d|^{2}-r_{1}^{2}\right)^{4}}+\frac{3 \beta^{2} r_{1}^{3} r_{2}^{3}\left(3|d|^{2}-r_{1}^{2}-r_{2}^{2}\right)}{\left(|d|^{2}-r_{1}^{2}-r_{2}^{2}\right)^{4}} \\
& K_{2}=1+\frac{\beta r_{1}^{3}|d|^{3}}{2\left(|d|^{2}-r_{2}^{2}\right)^{3}}+\frac{\beta r_{2}^{3}|d|^{3}}{2\left(|d|^{2}-r_{1}^{2}\right)^{3}}+\frac{3 \beta^{2} r_{1}^{3} r_{2}^{3}}{\left(|d|^{2}-r_{1}^{2}-r_{2}^{2}\right)^{3}}
\end{aligned}
$$

For small gaps between two droplets there is the filmthinning force acting on droplet 1 . Then the droplet-droplet force consists of the film-thinning force and the dipole-dipole electrically induced force (dipole-induced-dipole model), and can be expressed as follows:

$$
\begin{aligned}
F_{\mathrm{d}-\mathrm{d}} & =F_{\mathrm{r}}-F_{\mathrm{f}} \\
& =12 \pi \beta^{2} \varepsilon_{\mathrm{o}}|E|^{2} r_{2}^{3} r_{1}^{3}|d|^{-4}\left(3 K_{1}-1\right)-\frac{6 \pi \mu_{\mathrm{c}} r^{2} v}{h}
\end{aligned}
$$

\subsubsection{Theoretical analysis of the relative movement of two droplets}

According to above force analysis, Eq. (7) can be simplified as:

$$
m_{1} \frac{\mathrm{d} v_{1}}{\mathrm{~d} t}=F_{\mathrm{d}-\mathrm{d}}-F_{\mathrm{d}}
$$

When droplet 1 is very far away from droplet $2(h>>r)$, Eq. (17) can be derived as:

$$
\frac{\mathrm{d} v}{\mathrm{~d} t}=\frac{18 \beta^{2} \varepsilon_{\mathrm{o}}|E|^{2} r_{2}^{3}|d|^{-4}}{\rho_{\mathrm{d}}}-\frac{9 \mu_{\mathrm{c}} \rho_{\mathrm{c}}}{2 \rho_{\mathrm{d}}^{2} r_{1}^{2}} v
$$

Let

$$
\begin{aligned}
& f_{1}=\frac{18 \beta^{2} \varepsilon_{\mathrm{o}}|E|^{2} r_{2}^{3}|d|^{-4}}{\rho_{\mathrm{d}}} \\
& f_{2}=-\frac{9 \mu_{\mathrm{c}} \rho_{\mathrm{c}}}{2 \rho_{\mathrm{d}}^{2} r_{1}^{2}}
\end{aligned}
$$

It should be noted that the coefficient $f_{1}$ is concerned with the center-to-center distance $d$, so it is a time dependent variable, which is shown as $f_{1}=\frac{18 \beta^{2} \varepsilon_{\mathrm{o}}|E|^{2} r_{2}^{3}\left|d_{0}-v t\right|^{-4}}{\rho_{\mathrm{d}}}$, where $d_{0}$ 
represents the initial center-to-center distance before applying the electric field. In order to simplify the integration process of Eq. (18), the movement process in the time increment $\Delta t$ is investigated, which means that in the time increment $\Delta t$, the center distance can be considered as a fixed value and $f_{1}$ can be seen as a coefficient independent of time. Then the movement of the water droplets in the time increment can be achieved. This indicated that the displacement of droplet 1 is consistent with the explinear function after integrating Eq. (18) twice as as follows:

$$
x=A \mathrm{e}^{-t / B}+C t+D
$$

with

$$
\begin{aligned}
& A=\frac{Z_{1}}{f_{2}^{2}} \\
& B=-\frac{1}{f_{2}} \\
& C=-\frac{f_{1}}{f_{2}}
\end{aligned}
$$

$$
D=Z_{2}
$$

where $Z_{1}, Z_{2}$ are integration constants. The coefficients $A, B$, $C$ and $D$ are dependent on the diameters of the droplets, the center-to-center distance, the density and permittivity of the dispersed phase and the continuous phase, the viscosity of the continuous phase and the applied electric field.

When two droplets get closer $(h<r)$, the following expression is achieved:

$$
\frac{\mathrm{d} v}{\mathrm{~d} t}=\frac{9 \beta^{2} \varepsilon_{\mathrm{o}}|E|^{2} r_{2}^{3}|d|^{-4}\left(3 K_{1}-1\right)}{\rho_{\mathrm{d}}}-\left(\frac{9 \mu_{\mathrm{c}} r^{2}}{2 h \rho_{\mathrm{d}} r_{1}^{3}}+\frac{9 \mu_{\mathrm{c}} \rho_{\mathrm{c}}}{2 \rho_{\mathrm{d}}^{2} r_{1}^{2}}\right) v
$$

Let

$$
\begin{aligned}
& f_{3}=\frac{9 \beta^{2} \varepsilon_{\mathrm{o}}|E|^{2} r_{2}^{3}|d|^{-4}\left(3 K_{1}-1\right)}{\rho_{\mathrm{d}}} \\
& f_{4}=-\left(\frac{9 \mu_{\mathrm{c}} r^{2}}{2 h \rho_{\mathrm{d}} r_{1}^{3}}+\frac{9 \mu_{\mathrm{c}} \rho_{\mathrm{c}}}{2 \rho_{\mathrm{d}}^{2} r_{1}^{2}}\right)
\end{aligned}
$$

In order to simplify the calculation and reduce the error, the coefficient $f_{3}$ is considered as independent of time, and the movement in each time increment is investigated. After integrating of Eq. (20) twice, the displacement expression of droplet 1 is achieved as follows:

$$
\begin{aligned}
& x=E \mathrm{e}^{-t / F}+G t+H \\
& E=\frac{Z_{3}}{f_{4}^{2}} \\
& F=-\frac{1}{f_{4}} \\
& G=-\frac{f_{3}}{f_{4}}
\end{aligned}
$$

where $Z_{3}$ and $Z_{4}$ are integration constants. The coefficients
$E, F, G$ and $H$ are dependent on coefficient $K_{1}$, the diameters of droplets, the center-to-center distance, the density and permittivity of the dispersed phase and the continuous phase, the viscosity of the continuous phase and the applied electric field.

According to above analysis, under the applied electric field, the displacement of the droplet changes with time and the relationship is $x=A \mathrm{e}^{-t / B}+C t+D$. However, just for the different center-to-center distances and different forces acting on the droplets, the coefficients in the formulas are different. For the same size droplets, the displacements of droplet 1 and 2 both exhibit the same relationship $x=A \mathrm{e}^{-t / B}+C t+D$. During the relative movement process, the center-to-center distance of two droplets also changes with the explinear function.

In present work, the relative movement of two droplets in the applied electric field is mainly investigated, and to the above analysis indicates that: if the movement process of two droplets is divided into multiple parts, in each time increment the center-to-center distances all change with the explinear function $x=A \mathrm{e}^{-t / B}+C t+D$. Our proposed model is also verified through experimental study.

\section{Experimental method}

\subsection{Experimental facility}

The micro-experimental setup used for investigation is shown in Fig. 3.

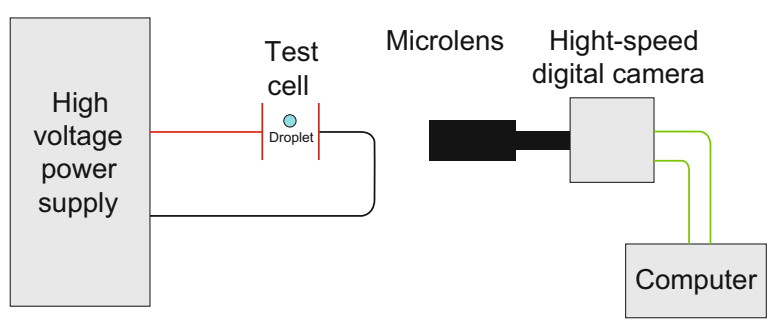

Fig. 3 Schematic diagram of the experimental system

The rectangular Perspex cell was $10 \mathrm{~mm} \times 20 \mathrm{~mm} \times 70 \mathrm{~mm}$ (Fig. 4), and two thin copper plates $(24 \mathrm{~mm} \times 70 \mathrm{~mm})$ were selected as the electrodes. The electrodes were fixed on opposite sides of the cell, and connected to a high voltage power supply. The temperature was $20{ }^{\circ} \mathrm{C}$ during experiment. A sinusoidal AC (voltage from -20 to $20 \mathrm{kV}$ and frequencies $50-400 \mathrm{~Hz}$ ) was generated by the high voltage power supply. The deformation and movement of emulsion drops in the AC electric field was imaged with a high speed camera (Fig. 5 NAC Hotshot) equipped with microscopic lenses and continuously recorded by a computer. The total optical magnification was 120 .

Three types of white oil and distilled water were chosen as the continuous phase and dispersed phase, respectively. For small drops, deformation can be ignored, so the water droplet is rather larger when studying drop deformation. During droplet coalescence, the droplet diameter is smaller than 200 $\mu \mathrm{m}$ and the angle between the center line of two droplets and the direction of the applied electric field is about $0^{\circ}$. The properties of white oil and distilled water are presented in 


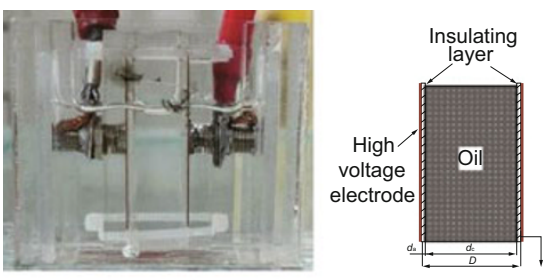

Fig. 4 Experimental cell

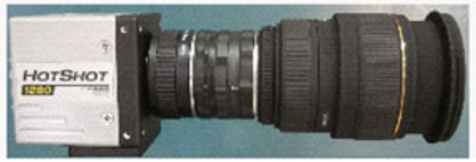

Fig. 5 High-speed digital camera

Table 1. In order to investigate the influence of interfacial tension, surfactant SDBS (sodium dodecyl benzene sulfonate) was added to change the interfacial tension. The amount and interfacial tension is shown in Table 2.

Table 1 Properties of the three oils and water at $15^{\circ} \mathrm{C}$

\begin{tabular}{cccc}
\hline Medium & $\begin{array}{c}\text { Viscosity } \\
\mathrm{mPa} \cdot \mathrm{s}\end{array}$ & $\begin{array}{c}\text { Relative dielectric } \\
\text { constant }\end{array}$ & $\begin{array}{c}\text { Surface tension } \\
\mathrm{mN} \cdot \mathrm{m}^{-1}\end{array}$ \\
\hline 1\# white oil & 2160 & 2.26 & 31.5 \\
2\# white oil & 785 & 2.24 & 31.7 \\
3\# white oil & 303 & 2.23 & 31.7 \\
Distilled water & 1.71 & 82 & 72.9 \\
\hline
\end{tabular}

Table 2 Interfacial tension of 1 \# white oil and water when SDBS surfactant is added

\begin{tabular}{cc}
\hline Content of SDBS, ppm & $\begin{array}{c}\text { Interfacial tension } \\
\sigma, \mathrm{mN} \cdot \mathrm{m}^{-1}\end{array}$ \\
\hline 0 & 41.1 \\
60 & 26.7 \\
100 & 10.8 \\
400 & 5.52 \\
\hline
\end{tabular}

\subsection{Method for analyzing drop images}

The resolution of images from the high speed camera is $1,280 \times 1,024$. Before the experiment, the camera images were calibrated at $2.84 \mu \mathrm{m} /$ pixel. Image-Pro Plus was used to manipulate the captured images. With this software, images can be processed by filtering, fast Fourier transform (FFT) and background correction in order to optimize the quality. In the image, we can choose measurement ranges and measure object properties, such as distance, area, center, angle, circle length, long axis and short axis. Also we can choose automatic or manual mode to calculate other properties, such as vibration frequency, deformation degree, relative velocity, movement trail and so on.

\section{Results and discussion}

\subsection{Deformation degree of the drop}

Under the AC electric field, droplets are influenced by the electric force and will oscillate periodically. In this paper the eccentricity of droplets is taken as the deformation degree of droplets. The difference of the maximum and minimum eccentricity is defined as the oscillation amplitude.

In Fig. 6, the diameter of the drop is about $866 \mu \mathrm{m}$, the electric field intensity is $8.4 \times 10^{5} \mathrm{~V} \cdot \mathrm{m}^{-1}$ and the frequency is $50 \mathrm{~Hz}$. The deformation degree increases gradually with time, and reaches a maximum value at $6 \mathrm{~ms}$. After that the deformation degree reduces gradually, and at $10 \mathrm{~ms}$, it reduces to the degree as it is at $0 \mathrm{~ms}$. During the experiment, droplets of different sizes give the same variation characteristics at a frequency of $50 \mathrm{~Hz}$, which is to say the deformation period is about $10 \mathrm{~ms}$. That means the frequency is $100 \mathrm{~Hz}$, which is twice the frequency of the electric field.

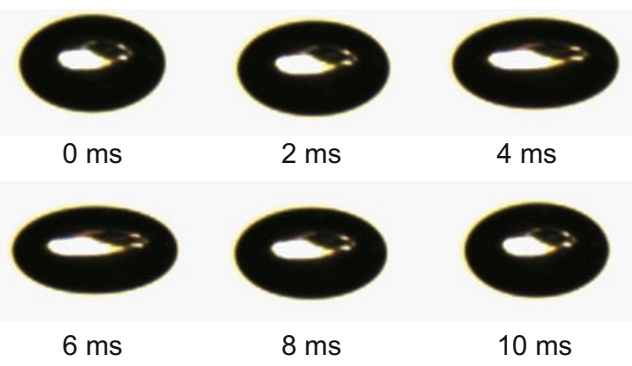

Fig. 6 Deformation of drop in the electric field

\subsubsection{The influence of the frequency}

Fig. 7 shows the average eccentricity of the droplets at different frequencies. The oscillation period of the drop is different under various electric field frequencies. The curve of the drop deformation degree is like a sine curve. Under different electric field frequencies, the frequency of eccentricity is about $100,200,400$, and $600 \mathrm{~Hz}$ respectively, which is about twice the electric field frequency. This is associated with the waveform of the sinusoidal AC electric field. As can be seen from Eq. (2), the electric force is proportional to the square of the electric field intensity, therefore, even if the electric field changes, the electric field intensity at a certain point of the drop does not change. Therefore, the drop will undergo an oscillation period during a half period of the $\mathrm{AC}$ electric field, which results in the oscillation frequency of the drop being twice the electric field frequency.

At different frequencies, the average eccentricity is about 0.63 , meaning that the average deformation degree is not influenced by the electric field frequency when the diameter is almost the same. For the average deformation degree is mainly influenced by the electric field intensity, and not influenced by frequency. But the oscillation amplitude of deformation degree is influenced by frequency, and it decreases gradually with an increase in the electric field frequency. This is because the period will decrease with an increase in frequency, thus the time during which the electric field intensity is above RMS will be reduced. So the drop is not fully stretched when the frequency increases, therefore, the oscillation amplitude will decrease.

\subsubsection{Influence of oil viscosity}

Drop deformation will be resisted by drag force $F_{\mathrm{d}}$ from oil. By using 1\#, 2\# and 3\# white oil, the deformation 

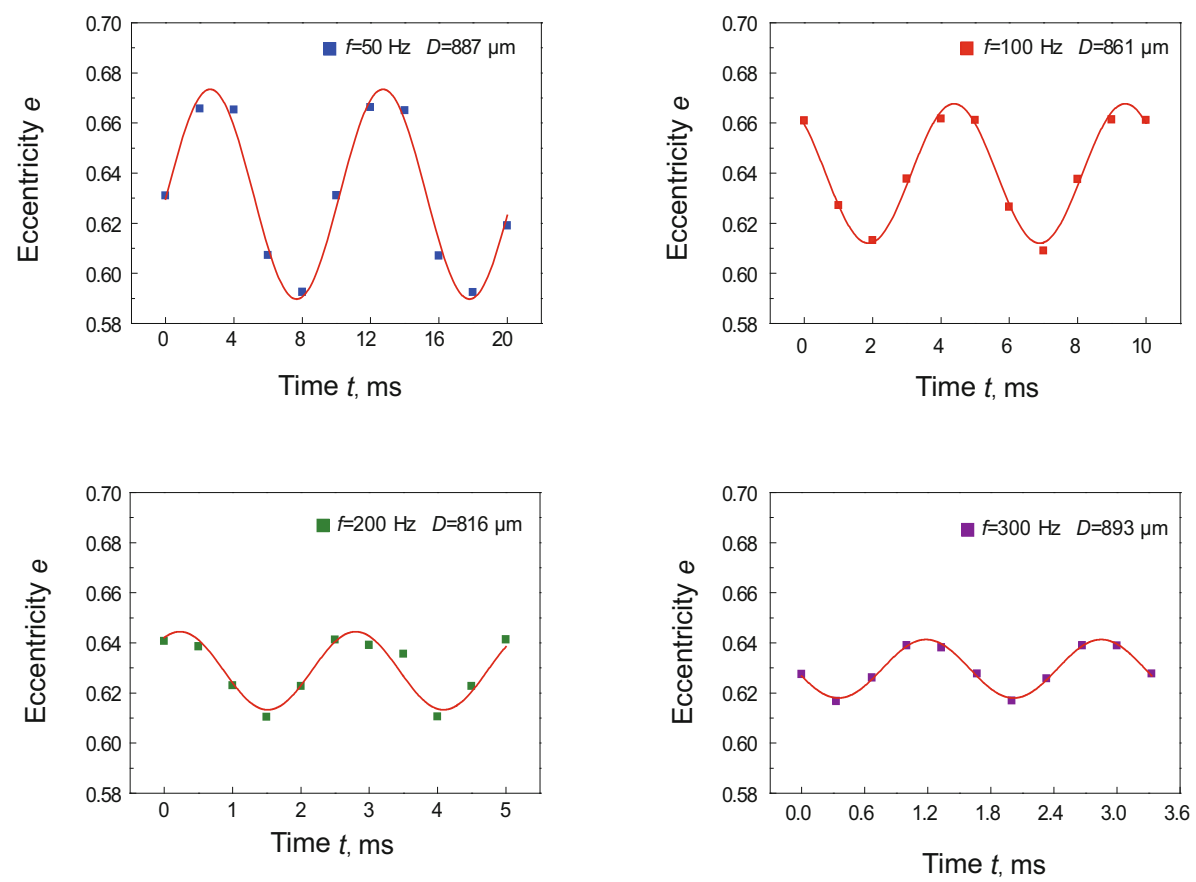

Fig. 7 Drop eccentricity changes with time in the electric field at different frequencies

characteristics of distilled water droplets in different oils were investigated.

Eow et al (2003) found that the deformation degree is only influenced by electrical properties in the DC electric field. In the AC electric field, the deformation degree changes during a cycle. We take the average deformation degree of one cycle as a factor to investigate. For 3 types of white oil, the dielectric constant is almost the same. It can also be found from Fig. 8, the average deformation degree of the drop is not so significantly influenced by oil viscosity for the average deformation degree of the drop is related to the electrical properties of oil but not related to oil viscosity. Drag force caused by oil will inhibit deformation when the drop deforms or moves. Therefore, the oil viscosity influences the oscillation amplitude of the droplet, but does not affect the average deformation degree.

The oscillation amplitude decreases with an increase in oil viscosity at the same electric field intensity. The viscosity of $3 \#$ white oil is the smallest. The eccentricity of the drop with a diameter of $866 \mu \mathrm{m}$ ranges from 0.57 to 0.69 , while for the drop with a diameter of $994 \mu \mathrm{m}$ its value ranges from 0.56 to 0.69 . In $2 \#$ white oil, the eccentricity of droplets, whose diameters are 887 and $979 \mu \mathrm{m}$, range separately from 0.59 to 0.67 and from 0.60 to 0.65 . The oscillation amplitude of the droplet in $1 \#$ white oil with the highest viscosity is the smallest.

Deformation of the droplet will be obstructed by the oil drag force. The higher the viscosity is, the bigger the drag force is. The interfacial tension among three types of oil and distilled water is almost the same, so the additional stress is almost the same. When the drag force is higher, the resistance is also higher. Then the deformation rate will reduce, as a result at the same time the oscillation amplitude will also reduce.


Fig. 8 Drop eccentricity changes with time in 1\#, 2\# and 3\# white oil

\subsubsection{Influence of the electric field intensity}

Fig. 9 shows the eccentricity of a distilled water drop with a diameter of $866 \mu \mathrm{m}$ under different electric field intensities in 3\# white oil when the electric field frequency is $50 \mathrm{~Hz}$. From Fig. 9, it is easy to see that with an increase in the electric field intensity, the average deformation degree increases gradually, but the oscillation amplitudes under different electric field intensities are all about 0.15 . This means the variation of eccentricity under different electric field intensities is almost the same in a given dispersion system.

With an increase in the electric field intensity, the electric force $F_{\mathrm{e}}$ increases, so the droplet deformation degree gradually increases. In the two endpoints of the ellipsoid, the curvature is the maximum. According to Eqs. (2) and 




Fig. 9 Drop eccentricity changes with time at different electric field intensities

(4), the electric force and the additional pressure acting on both endpoints of the ellipsoid will increase. The additional pressure will prevent droplet deformation. When the electric field intensity increases, the increasing electric force elongates the drop, while the additional pressure prevents deformation. Therefore, under the combined effect of additional pressure and drag force, the increased electric force does not increase the deformation degree. At high electric field intensities, the droplet oscillation amplitude does not increase. However, if the electric field intensity exceeded a certain value, droplets would break, which is not conducive to droplet coalescence.

\subsubsection{The influence of the droplet diameter}

The eccentricity of 4 different droplets in $2 \#$ white oil under the same electric field is shown in Fig. 10. It can be seen that the drop deformation degree increases with an increase in droplet diameter. The reason is that the electric force increases with an increase in the droplet diameter. However, the variation of deformation degree for different droplets is the same, from 0.06 to 0.08 , suggesting that the influence of droplet diameter on oscillation amplitude can be ignored, which is relative to the interaction of additional pressure, electric force and drag force when the drop is deformed. The electric force and the deformation degree

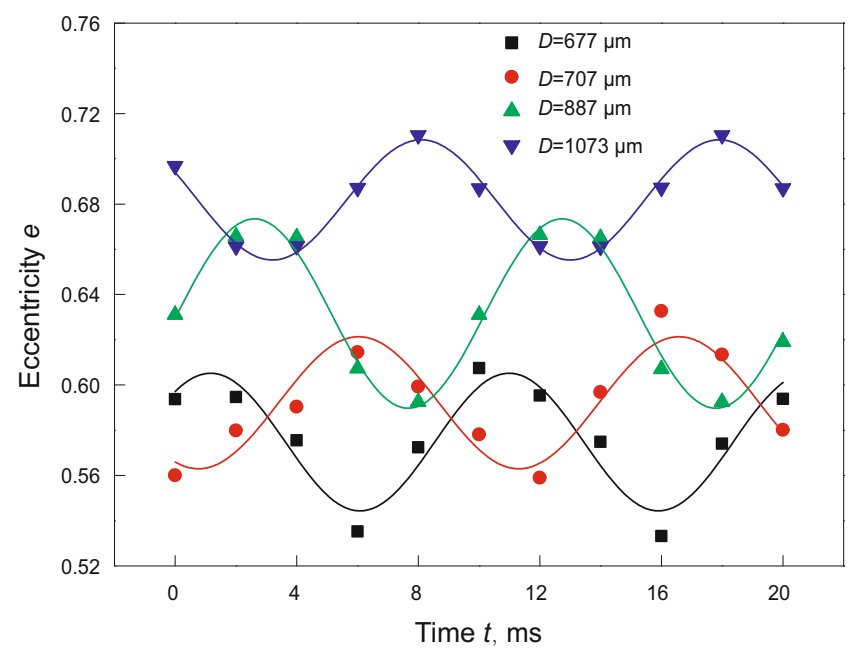

Fig. 10 Drop eccentricity changes with time for different diameter drops increase as the droplet diameter increases. In the two endpoints of the long axis, the curvature is the largest, so the electric force and the additional pressure is also the biggest. However, the increased droplet diameter can also increase drag force. Under the effect of additional pressure and drag force, the increase in the oscillation amplitude caused by increasing electric force can be inhibited. Therefore, the increase in the droplet diameter does not increase the deformation oscillation amplitude.

\subsection{Movement characteristics of double droplets}

The frequency and intensity of the applied electric field is $50 \mathrm{~Hz}$ and $4.36 \times 10^{5} \mathrm{~V} \cdot \mathrm{m}^{-1}$, respectively. The initial relative center-to-center distance of the two droplets is 2.1 , and the diameters are both $145 \mu \mathrm{m}$. The position and status of a 145 $\mu \mathrm{m}$ double droplet system in the electric field at different times are shown in Fig. 11. In order to compare, the time necessary for complete coalescence of two droplets is defined as time $0 \mathrm{~ms}$. After applying the AC electric field, the dipoledipole electrically induced force acting on the droplets can overcome the drag force and the droplets will attract each other and then make contact. After that, the drainage and rupture of the liquid film occurs, eventually completing the entire coalescence process.

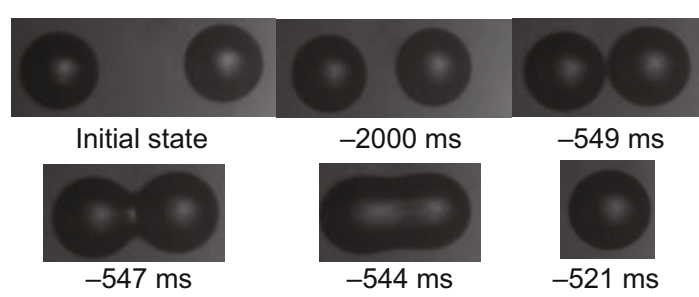

Fig. 11 States of $145 \mu \mathrm{m}$ double droplets in the applied electric field

\subsubsection{Two-segment fitting of the center-to-center distance between two droplets}

Fig. 12 shows the change curve and local enlarged view of the center-to-center distance between the two droplets at $6 \mathrm{~s}$ before coalescence. Fig. 12(a) indicates that the centerto-center distance gradually reduces and the mean velocity increases. The change of center-to-center distance at $1 \mathrm{~s}$ before coalescence is partially enlarged in Fig. 12(b). In the $\mathrm{AC}$ electric field, the intensity and direction of the electric field change with time, and then the polarized charge density, polarization and the electrically induced force all vary with the electric field. As a result, the droplets also oscillate while getting close to each other. By differentiating the center-tocenter distance between droplets as shown in Fig. 12(b), the instantaneous relative velocity of $145 \mu \mathrm{m}$ double droplets in the applied electric field is achieved in Fig. 13.

As shown in Fig. 13, negative velocity values represent that the droplets move towards each other and they become much closer, while positive velocity values indicate that due to the change of the electric field direction the droplets move apart. In addition, it is because the applied electric intensity varies with time that the absolute value of the velocity fluctuates. 


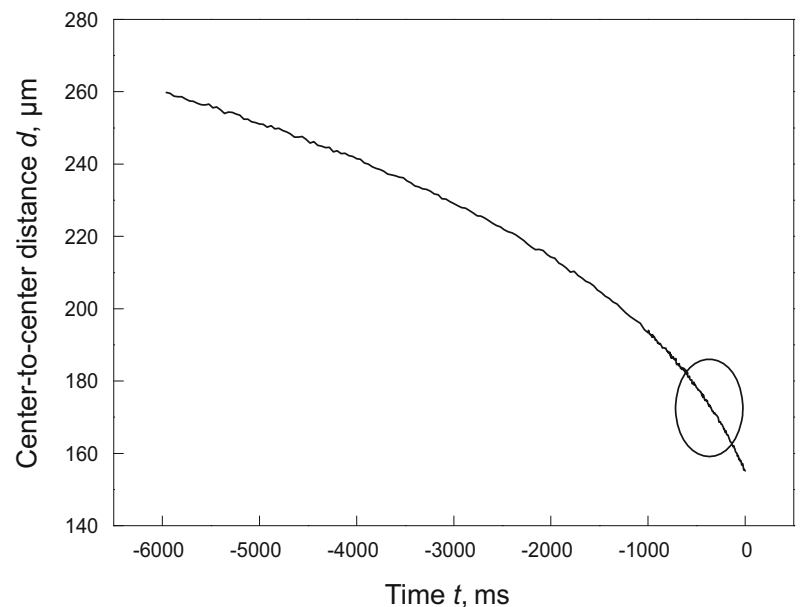

(a) Change of center-to-center distance with time

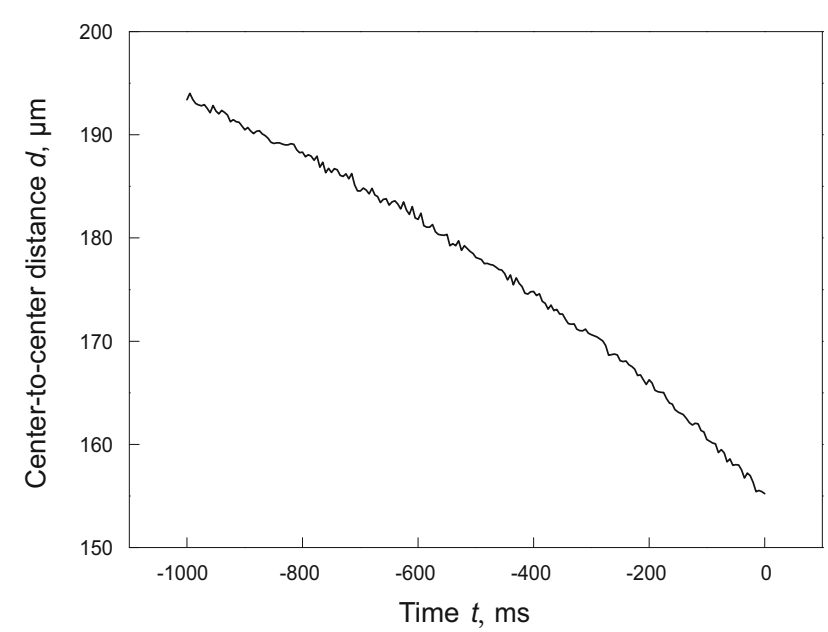

(b) Local enlarged view

Fig. 12 Change of the center-to-center distance between two droplets with time

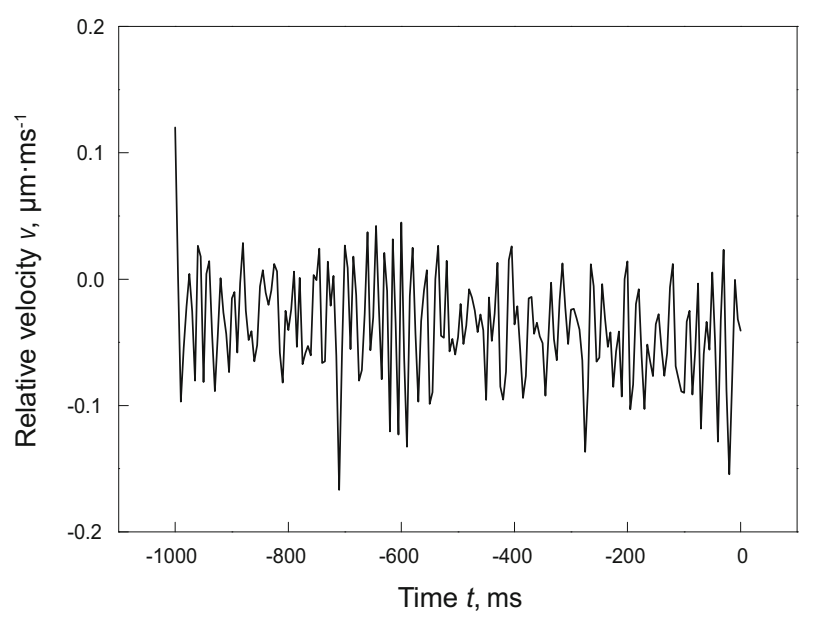

Fig. 13 The instantaneous relative velocity

According to theoretical analysis, if the movement process of double droplets is divided into several small parts, the center-to-center distance in each time increment changes with time: $x=A \mathrm{e}^{-t / B}+C t+D$. In present work, the relative movement process of $145 \mu \mathrm{m}$ droplets is divided into two parts: from -6 to $-1 \mathrm{~s}$ and from -1 to $0 \mathrm{~s}$. Fig. 14 shows a comparison between the experimental curve and fitted curve of the center-to-center distance between two droplets.

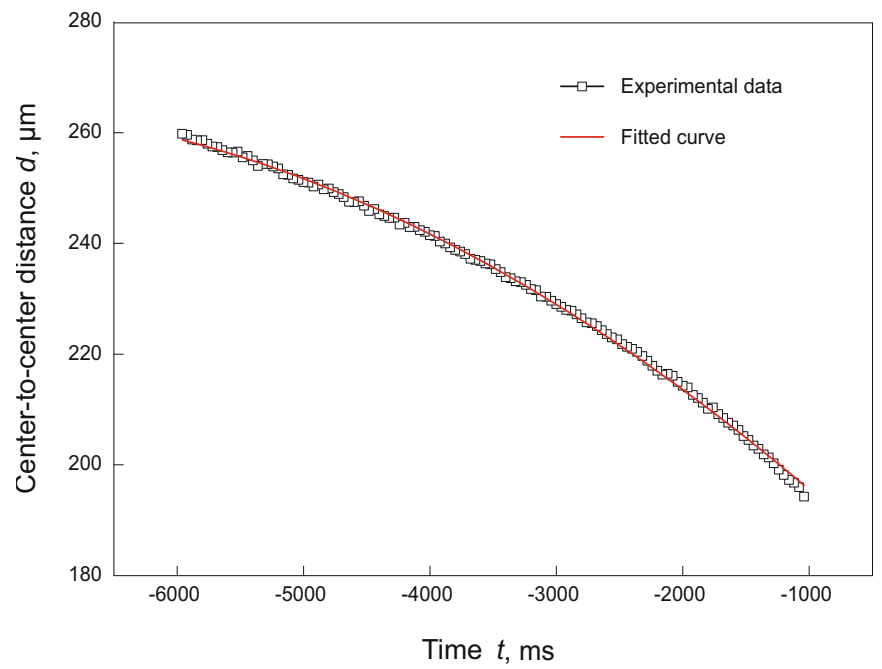

(a) Center-to-center distance at from 1 to $6 \mathrm{~s}$ before coalescence

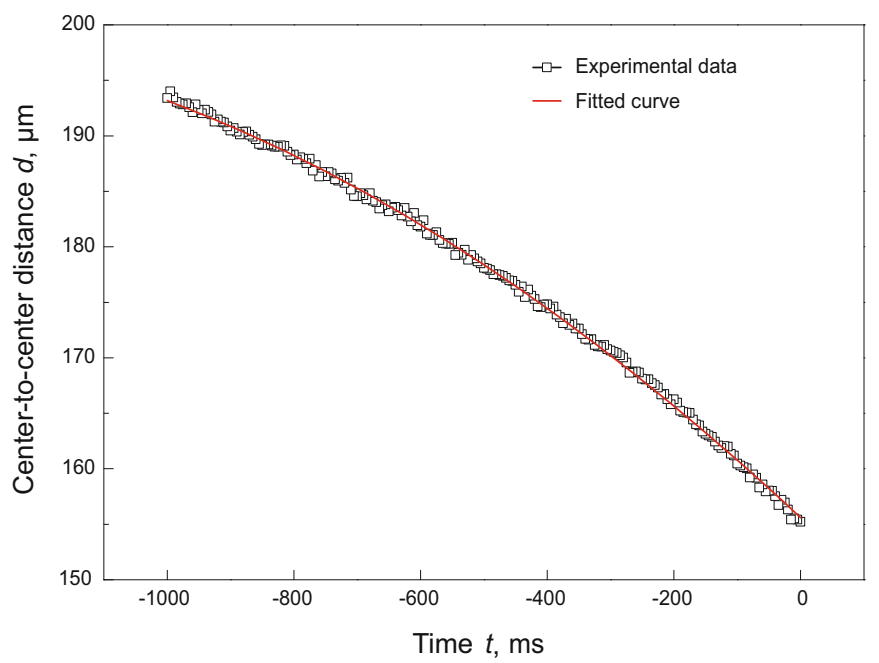

(b) Center-to-center distance at from 1 to $0 \mathrm{~s}$ before coalescence

Fig. 14 Experimental data and fitted curve of the center-to-center distance between two droplets

An explinear function is selected to fit experimental data. From -6 to $-1 \mathrm{~s}$ the expression $d=-7685.3 \mathrm{e}^{-t / 55140.0}-0.161 t+7860.28$ is achieved. After $-1 \mathrm{~s}$ the expression $d=-4995.13 \mathrm{e}^{-t / 12753.26}-0.445 t+5150.69$ is obtained. Two different fitted curves both show a good consistency. The differences of coefficients in the two relationships are mainly caused by the different forces acting on droplets at various stages. Eqs. (19) and (21) correspond to two different movement processes. As the center-tocenter distance between droplets decreases, the dipoledipole electrically induced force will sharply increase, so $f_{1}<f_{3}$. For a large center-to-center distance (from -6 to $-1 \mathrm{~s}$ ), when two droplets get closer the drag force will prevent them moving together. As the center-to-center distance further 
reduces (after $-1 \mathrm{~s}$ ), in addition to the drag force, there is also the film-thinning force acting on droplets to prevent them getting closer, so $\left|f_{2}\right|<\left|f_{4}\right|$, then in the fitting relationships $A>E$ and $B>F$ are achieved. With the decrease in the centerto-center distance, the film-thinning force and dipole-dipole electrically induced force both increase, but the rate of increase of the latter is much larger than that of the former and the electrically induced force can overcome the resistance of the film-thinning force, so $\left|\frac{f_{1}}{f_{2}}\right|<\left|\frac{f_{3}}{f_{4}}\right|$ and $C<G$. The two fitted curves corresponding to a time period of from -6 to -1 $\mathrm{s}$ and after $-1 \mathrm{~s}$ are consistent with the explinear function. According to theoretical analysis, if the movement process is divided into more segments, fitting of the experimental data is more similar to an explinear function.

As shown in Fig. 15, the mean relative velocity of 145 $\mu \mathrm{m}$ droplets can be estimated by taking the derivative of the fitted curve of the center-to-center distance in Fig. 14. This indicates that as the center-to-center distance decreases, the electrically induced force and acceleration greatly increase, as a result the mean relative velocity increases. As the approaching process is divided into two parts at -1 $\mathrm{s}$, a discontinuous point appears in the mean velocity curve. Therefore, if the movement process is divided into more segments, the number of discontinuous points will increase in this way. Although making use of explinear function to fit the movement process which is divided into several parts can achieve a good consistency, the obtained velocity curve is not continuous and can not facilitate the analysis of the whole movement process before coalescence.



Fig. 15 Average relative velocity of double droplets

\subsubsection{Using a fourth order polynomial to fit the movement process of double droplets}

In order to investigate the whole movement process before coalescence, a number of functions are selected to fit the center-to-center distance experimental data, and a fourth order polynomial relationship $x=a t^{4}+b t^{3}+c t^{2}+d t+e$ can give a good fit. Fig. 16(a) shows the experimental curve and fitted curve of the $145 \mu \mathrm{m}$ droplets.

As is shown in Fig. 16(a), the relative movement process of $145 \mu \mathrm{m}$ droplets in the $6 \mathrm{~s}$ before coalescence is fitted with a polynomial function $d=156.31-0.05 t-1.50 \times 10^{-5} t^{2}-2.67 \times 10^{-9} t^{3}$
$-1.81 \times 10^{-14} t^{4}$, and a good agreement is obtained. Fig. 16(b) shows the mean relative velocity of $145 \mu \mathrm{m}$ droplets. From -6 to $-1 \mathrm{~s}$, when the center-to-center distance is large, dipoledipole force (point-dipole model) and drag force both act on the droplet, and the relative movement is quite slow, but tends to accelerate. After $-1 \mathrm{~s}$, the center-to-center distance reduces rapidly. In addition to the dipole-dipole force there is also the film-thinning force. As the center-to-center distance further decreases, the dipole-dipole force increases rapidly, and is greater than the increasing drag force and film-thinning force, so the relative movement is quite fast, and also tends to accelerate. It should be noted that from -4.5 to $-2.5 \mathrm{~s}$, the average relative velocity is quite small, and tends to be constant. In this period, the relative center-to-center distance $h /(2 r)$ is from 0.5 to 1 , and the dipole-dipole force changes from the point-dipole model to the dipole-induced-dipole model. At the same time, the film-thinning force begins to act on droplets. Therefore, in this special period the dipoledipole force can overcome the sum of the drag force and film-thinning force, and then all the forces reach a relative balance. In other words, there is no acceleration in the relative movement, so the mean velocity tends to be constant.



(a) Change of center-to-center distance

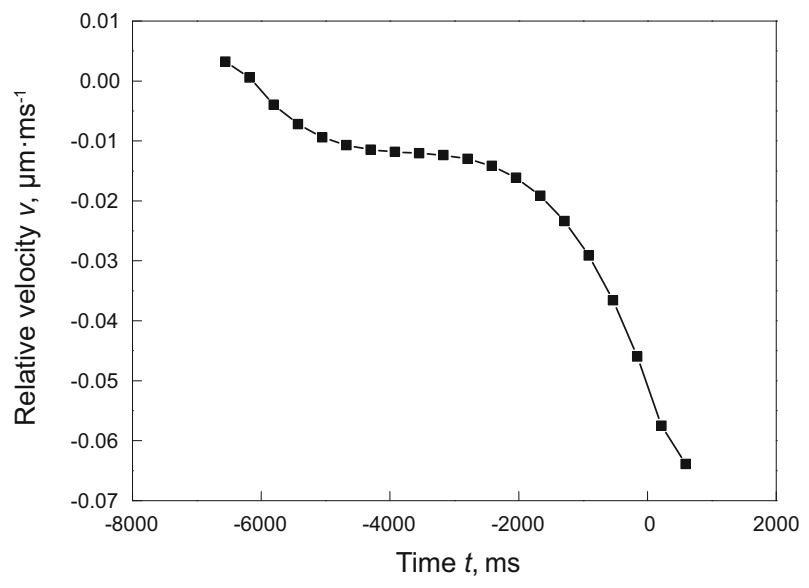

(b) Mean relative velocity

Fig. 16 Center-to-center distance and average relative velocity of double droplets 


\subsubsection{Influence of electric field intensity on movement velocity}

Usually the coalescence efficiency in water-in-oil emulsion increases with increasing electric field intensity. However, when the electric field intensity exceeds the limited strength of droplet-droplet coalescence, the electrically induced force will greatly increase the deformation of water droplets. Then the droplet will burst into smaller droplets, called the "electro-breakup" phenomenon. In the present micro-experiment, distilled water and $1 \#$ white oil are selected as the dispersed phase and the continuous phase. The frequency of the applied AC electric field is $50 \mathrm{~Hz}$, and the electric field intensity is not larger than the limiting value.

The initial relative center-to-center distance before applying electric field is 3.3. The relative velocity of $145 \mu \mathrm{m}$ double droplets in different electric field intensity is shown in Fig. 17. The two droplets attract and approach each other due to the electrical force acting on the droplets. According to Eq. (11), the dipole-dipole electrical force is proportional to the square of the electric field intensity. The micro-experimental data show that the dipole-dipole electrical force between two droplets increases significantly with increasing electric field intensity, which can accelerate the relative velocity of the droplets. In addition, five different curves of relative velocity show that the relative velocity increases significantly with the electric intensity.

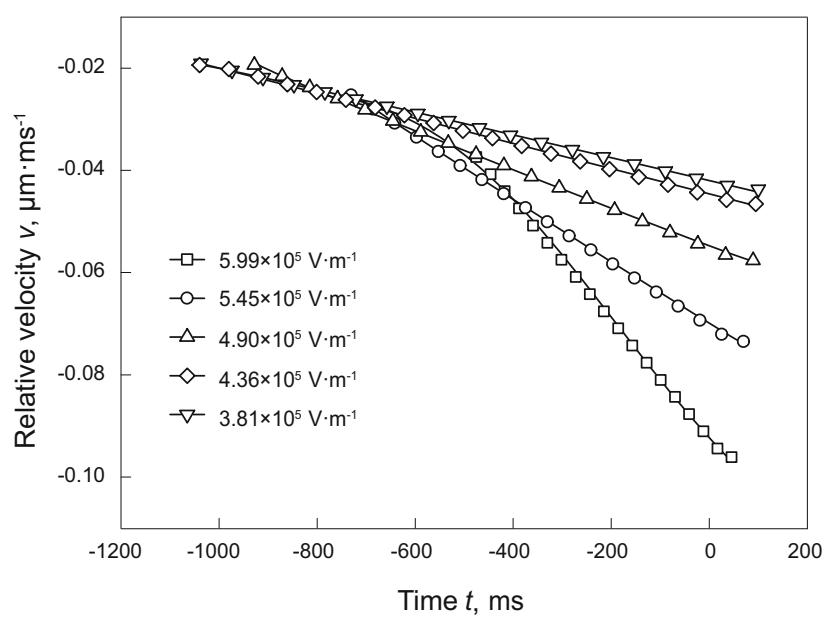

Fig. 17 Relative velocity at different electric fields

\subsubsection{Influence of the droplet diameter}

The frequency and intensity of the applied AC electric field are $50 \mathrm{~Hz}$ and $5.45 \times 10^{5} \mathrm{~V} \cdot \mathrm{m}^{-1}$. The five droplet pairs had the same initial relative center-to-center distance. The relative velocities of the five pairs of droplets (droplet diameter: 100, $125,140,165$ and $190 \mu \mathrm{m}$ ) in the $1 \mathrm{~s}$ before coalescence are shown in Fig. 18.

As is shown in Fig.18, the electrical force acting on the droplets may overcome the drag force and help them approach each other. With the decrease in center-to-center distance between two droplets, the average relative velocities of all the five groups of droplets increase. The relative velocity of the large droplets grows faster because under the same electric field the electrically induced force acting on a large droplet is larger than that on a smaller one. In other

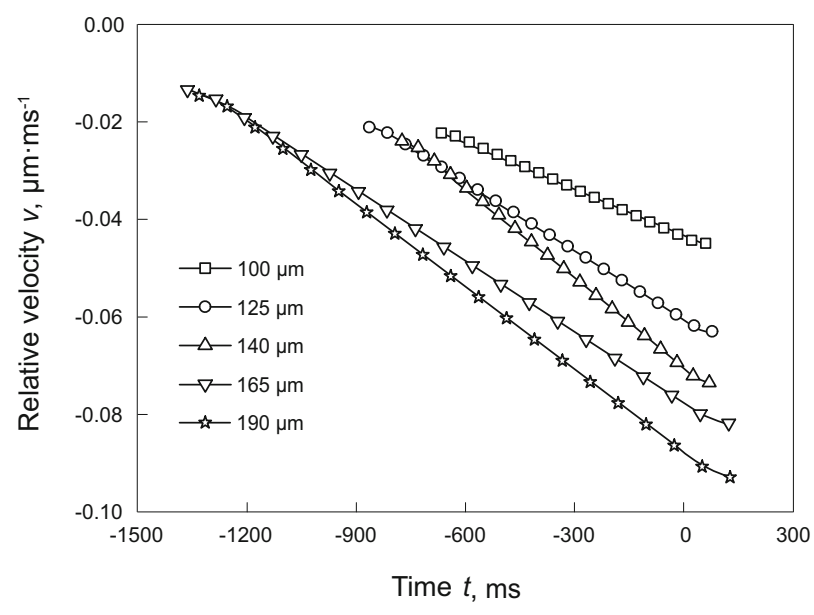

Fig. 18 Relative velocity of double droplets of different sizes

words, the relative velocity of the larger droplets is faster than that of the smaller ones. To some extent, it can reflect that the bigger water-doublets move faster than the smaller ones in the same applied electric field, so the larger droplets can be separated from the oil emulsion more quickly.

\subsubsection{The influence of oil viscosity}

Three different pairs of droplets, with diameter of 220 170 , and $140 \mu \mathrm{m}$, respectively, are suspended in two samples of oil of different viscosities. The electric field intensities are $4.36 \times 10^{5}, 4.90 \times 10^{5}$, and $5.45 \times 10^{5} \mathrm{~V} \cdot \mathrm{m}^{-1}$. The relative velocity of the double droplets in the $1 \mathrm{~s}$ before coalescence is shown in Fig. 19.

Fig. 19 show that the relative velocity curves of different double droplets in different oil differ a lot. In more viscous oil, the water droplets move quite more slowly. During movement of the water droplet in the applied electric field, besides the electrical force, the drag force and the filmthinning force are both affected by the viscosity of the continuous phase. Taking the $220 \mu \mathrm{m}$ water droplets as an example, under the same electric field when the center-tocenter distances are the same the electrically induced forces in different oil are approximately the same. However, in more viscous oil, the drag force and the film-thinning force preventing the droplets to approach each other are much larger than those in the other oil. Therefore, due to the larger resistance force acting on droplets in more viscous $1 \#$ white oil, the acceleration is much smaller and the relative velocity is much lower. As the viscosity of $2 \#$ white oil is quite low, then water droplets move more quickly.

\subsubsection{The influence of interfacial tension}

Natural emulsifying agents consisting of aliphatic acids, asphaltene and wax exist on the oil-water interface, hindering droplet coalescence. The existing emulsifying agents make the interfacial film very stable and produce a physical barrier to droplet coalescence for the dehydration of crude oil. In order to investigate the effect of interfacial tension on droplet coalescence, SDBS is chosen to prepare solutions of different surfactant concentrations $(10,60,100$, and $400 \mathrm{ppm})$ with distilled water as the reference dispersed phase.

The frequency and electric field intensity are $50 \mathrm{~Hz}$ and $3.81 \times 10^{5} \mathrm{~V} \cdot \mathrm{m}^{-1}$. SDBS solutions of different concentrations are chosen as the dispersed phase to prepare five pairs of 180 


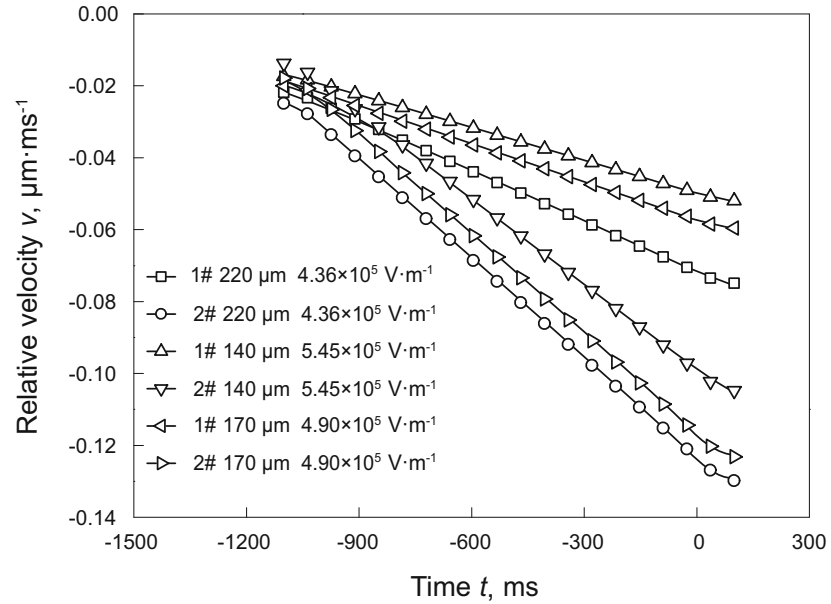

Fig. 19 Relative velocity of double droplets in \#1 and 2\# white oil (viscosities 2160 and $785 \mathrm{mPa} \mathrm{s}$, respectively)

$\mu \mathrm{m}$ double droplets. The initial center-to-center distance of the droplets were the same. The relative velocities of five pairs of $180 \mu \mathrm{m}$ double droplets in the $3 \mathrm{~s}$ before coalescence are shown in Fig. 20(a). The interfacial tension between distilled water and $1 \#$ white oil is the largest (Table 2), so the ability to resist deformation caused by electric field is the strongest. Therefore, the deformation of distilled water droplets is the smallest, and the polarization is weakened, then the velocity towards each other is slow. Fig. 20(a) indicates that under the same electric field, there is little significant difference in the relative velocities of water droplets containing SDBS (10$400 \mathrm{ppm}$ ). When the frequency and electric field intensity are $50 \mathrm{~Hz}$ and $4.36 \times 10^{5} \mathrm{~V} \cdot \mathrm{m}^{-1}$, the relative velocities of five pairs of $150 \mu \mathrm{m}$ double droplets are shown in Fig. 20(b). For smaller droplets the interfacial tension has little effect on the relative velocity because the droplets tend to be rigid and the interfacial tension decreases with the decreasing diameter.

\subsection{Movement characteristics of droplets in emulsion}

The coalescence characteristics of little droplets in $2 \#$ white oil under the AC electric field are studied with the high speed microscopic camera system. Most of the droplets are less than $100 \mu \mathrm{m}$, which is much smaller than the droplets used during previous experiments (Eow et al, 2001; 2003; Eow and Ghadiri, 2003b). As shown in Fig. 21, at first the nearby droplets in area 1 begin dipole coalescence to form large droplets after the electric field is applied for $67 \mathrm{~ms}$. The large droplets attract small ones more easily to form even larger droplets. After dipole coalescence droplets are easier to move together to form short droplet chains. As shown in areas 2 and 3 , there are only 3 droplets in a short chain. When short chains come close to each other, they may attract each other under the effect of electric force and form longer droplet chains. Short chains in areas 2 and 4 form longer chains in area 5, at the same time droplets at the end of the chain coalesce when the chains get closer. As shown in Fig. 21(k), the chains in area 5 can attract small droplets around them. Also in Fig. 21(1), the droplets in chains can also coalesce to form larger droplets. Thus there are 3 types drop coalescence in chain coalescence. These are 1) coalescence within

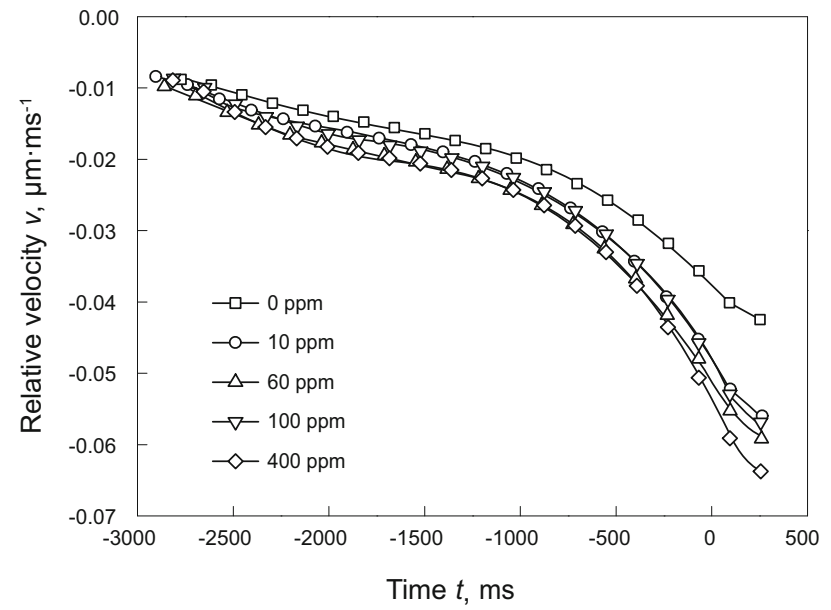

(a) Relative velocity of the $180 \mu \mathrm{m}$ droplets

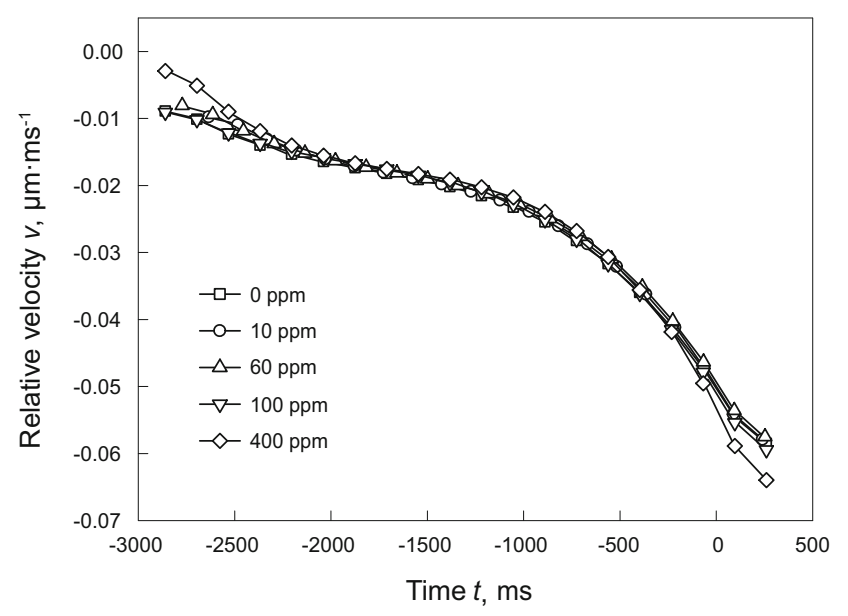

(b) Relative velocity of the $150 \mu \mathrm{m}$ droplets

Fig. 20 The relative velocity of $180 \mu \mathrm{m}$ and $150 \mu \mathrm{m}$ double droplets with different surface tensions

chains, 2) coalescence between chains and 3) coalescence by attracting small nearby droplets.

By comparing the droplets of diameter $200 \mu \mathrm{m}$ in Fig. 21(f) and (j), it can be found the increase in electric field intensity deforms the droplets to be ellipsoidal after chains is formed. The deformation degree of a drop in a chain is 0.43 , while a drop with the same diameter not in the chain do not deform and is like a rigid sphere. The higher electric field intensity in the chains leads to the coalescence of small nearby droplets.

\section{Conclusions}

1) In a AC electric field, the average degree of deformation of droplets increases with an increase in the electric field intensity and droplet diameter. The electric frequency and oil viscosity have no significant influence.

2) The amplitude of deformation oscillation is mainly affected by electric field frequency and oil viscosity. High frequency and high oil viscosity will decrease the oscillation amplitude. Electric field intensity and droplet diameter have no obvious influence on oscillation amplitude.

3) The forces acting on a double droplet system in an AC 


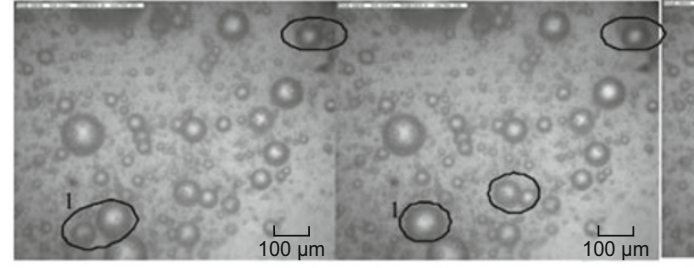

(a) $0 \mathrm{~ms}$

(b) $66.67 \mathrm{~ms}$

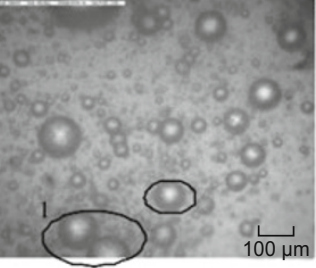

(c) $400 \mathrm{~ms}$

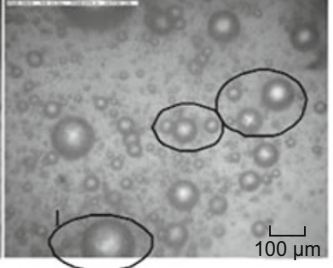

(d) $433.33 \mathrm{~ms}$

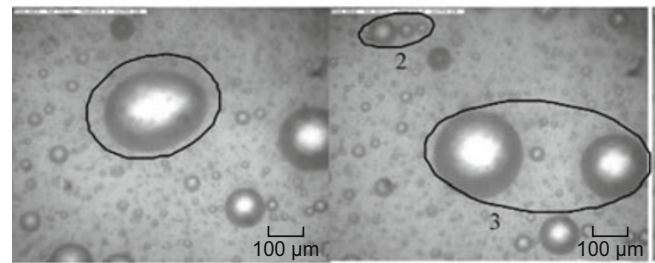

(e) $11783.33 \mathrm{~ms}$ (f) $16033.33 \mathrm{~ms}$



(g) $20333.33 \mathrm{~ms}$

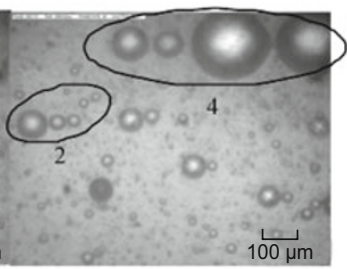

(h) $28950 \mathrm{~ms}$



(i) $32550 \mathrm{~ms}$

(j) $33716.67 \mathrm{~ms}$

(k) $36966.67 \mathrm{~ms}$

(I) $37000 \mathrm{~ms}$

Fig. 21 Changes of drops with time $\left(f=100 \mathrm{~Hz}, E=500 \mathrm{kV} \cdot \mathrm{m}^{-1}\right)$

electric field are analyzed theoretically. For large center-tocenter distances, there are mainly dipole-dipole electrically induced force and drag force acting on the droplet in the horizontal direction. For small center-to-center distances, in addition to the dipole-dipole force and drag force, there is also the film-thinning force to inhibiting droplets from getting close. Combined with Newton's second law, the force expressions are simplified and derived. This indicates that if the relative movement is divided into several small segments, the center-to-center distances in each part can be fitted using an explinear function. The more segments into which the movement process is divided, the smaller the error is.

4) A micro-experiment is conducted to investigate the movement and coalescence of $145 \mu \mathrm{m}$ double horizontal droplets in an applied AC electric field. Based on analyzing the center position of droplets at different times, the variation of the center-to-center distance between the droplets and the instantaneous velocity of relative movement can be measured. The movement in the 6 seconds before coalescence is divided into two parts: from -6 to $-1 \mathrm{~s}$ and from -1 to $0 \mathrm{~s}$. Using an explinear function to fit each partial movement process obtains a good fit. In order to investigate the whole movement process of water droplets a fourth order polynomial $x=a t^{4}+$ $b t^{3}+c t^{2}+d t+e$ is proposed to fit the center distance between two droplets, and a good fit is achieved.

5) The applied electric field is the main driving force to promote droplet coalescence. A high electric field intensity can significantly increase the relative droplet velocity. In an appropriate range of electric field intensity, increasing the intensity can improve the efficiency of electrostatic coalescence. Under the same electric field, the relative velocity of big droplets is faster than that of small ones. The oil viscosity affects relative velocity due to the drag force.
High viscosity of oil decreases the movement velocity. Only when the interfacial tension is high, can it influence the droplet movement velocity and for smaller droplets the influence of interfacial tension will be weakened.

6) From the micro scale experiment, it can be found that dipole coalescence and chain coalescence mainly occur under the AC electric field. After dipole coalescence, large droplets may form chains. There are 3 types of droplet coalescence in chains.

\section{Acknowledgements}

The work is partially supported by a grant from Chinese National Natural Science Foundation (Grant Nos. 51106182, 51274233, 51006124) and Natural Science Foundation of Shandong Province of China (ZR2010EQ040).

\section{References}

Bailes P J and Larkai S K L. An experimental investigation into the use of high voltage DC field for liquid phase separation. Transactions of the Institution of Chemical Engineers. 1981. 59(4): 229-237

Bailes P J and Larkai S K L. Liquid phase separation in pulsed D.C. fields. Transactions of the Institution of Chemical Engineers. 1982. 60(2): 115-121

Bailes P J and Stitt E H. Column liquid contacting with vigorous agitation balanced by electrostatic coalescence. Chemical Engineering Research and Design. 1987. 65(1): 514-523

Chen T Y, Mohammed R A and Bailey A I. Dewatering of crude oil emulsions 4. Emulsion resolution by the application of an electric field. Colloids and Surfaces A: Physicochemical Engineering Aspects. 1994. 83(3): 273-284

Chiesa M, Melheim J A and Hannisdal A. Emulsion of heavy crude oils, influence of viscosity, temperature and dilution. Journal of Dispersion Science and Technology. 2005a. 26(5): 615-627 
Chiesa M, Melheim J A, Pedersen A, et al. Forces acting on water droplets falling in oil under the influence of an electric field: numerical predictions versus experimental observations. European Journal of Mechanics-B/Fluids. 2005b. 24(6): 717-732

Chiesa M, Ingebrigtsen S and Melheim J A. Investigation of the role of viscosity on electrocoalescence of water droplets in oil. Separation and Purification Technology. 2006. 50(2): 267-277

Davis R H, Schonberg J A and Rallison J M. The lubrication force between two viscous drops. Physics of Fluids A. 1989. 1(1): 77-81

Eow J S and Ghadiri M. Electrostatic enhancement of coalescence of water droplets in oil: a review of the current understanding. Chemical Engineering Journal. 2001. 84(3): 173-192

Eow J S, Ghadiri M and Sharif A. Deformation and break-up of aqueous drop in dielectric liquids in high electric fields. Journal of Electrostatics. 2001. 51-52(1): 463-469

Eow J S and Ghadiri M. Electrostatic enhancement of coalescence of water droplets in oil: a review of the technology. Chemical Engineering Journal. 2002. 85(2-3) 357-368

Eow J S and Ghadiri M. Drop-drop coalescence in an electric field: the effects of applied electric field and electrode geometry. Colloids and Surfaces A: Physicochemical Engineering Aspects. 2003a. 219(1): 253-279

Eow J S and Ghadiri M. Motion, deformation and break-up of aqueous drops in oils under high electric field strengths. Chemical Engineering and Processing. 2003b. 42(4): 259-272

Eow J S, Ghadiri M and Sharif A. Experimental studies of deformation and break-up of aqueous drops in high electric fields. Colloids and Surfaces A: Physicochemical and Engineering Aspects. 2003. 225(1-3): 193-210

Eow J S, Ghadiri M and Sharif A. Electro-hydrodynamic separation of aqueous drops from flowing viscous oil. Journal of Petroleum Science and Engineering. 2007. 55(1-2): 146-155

Feng S and Guo K. Oil \& Gas Gathering Transportation and Processing in Mines. Shandong: China University of Petroleum Press. 2006 (in Chinese)

Guo J. Charge distribution on the conductors ball surface in an external homogeneous electric field. Journal of Southwest University of
Science and Technology. 2001. 16(1): 66-68 (in Chinese)

Harpur G, Wayth N J, Bailey A. G et al. Destabilisation of water-in-oil emulsions under the influence of an A.C. electric field: experimental assessment of performance. Journal of Electrostatics. 1997. 40-41(1): $135-140$

Klingenberg D J, Swol F and Zukoski C F. The small shear rate response of electrorheological suspensions. II. extensions beyond the pointdipole limit. Journal of Chemical Physics. 1991. 94(9): 6170-6178

Siu Y L, Wan T K and Yu K W. Interparticle force in polydisperse electrorheological fluid: beyond the dipole approximation. Computer Physics Communications. 2001. 142(1): 446-452

Supeene G, Koch C R and Bhattacharjee S. Deformation of a droplet in an electric field: nonlinear transient response in perfect and leaky dielectric media. Journal of Colloid and Interface Science. 2008. 31(8): 463-476

Taylor S E. Investigations into the electrical and coalescence behaviour of water-in-crude oil emulsions in high voltage gradients. Colloids and Surfaces. 1988. 29(1): 29-51

Taylor S E. Theory and practice of electrically enhanced phase separation of water-in-oil emulsions. Transactions of the Institution of Chemical Engineers. 1996. 74(5): 526-540

Urdahl O, Berry P, Wayth N, et al. The development of a new compact electrostatic coalescer concept. SPE Annual Technical Conference and Exhibition, New Orleans, Louisiana, USA, 27-30 September 1998

Williams T J and Bailey A. G. Changes in the size distribution of a water-in-oil emulsion due to electric field induced coalescence. IEEE Transactions on Industry Applications. 1986. 22(3): 536-541

Williams T J. The Resolution of Water-in-oil Emulsion by Application of An External Field. Ph.D. Thesis. University of Southampton, UK. 1989

Zhang Y, Liu Y and Ji R. Dehydration efficiency of high-frequency pulsed DC electrical fields on water-in-oil emulsion. Colloids and Surfaces A: Physicochemical and Engineering Aspects. 2011. 373 (1-3): 130-137

(Edited by Sun Yanhua) 